Unterrichtsinhalte und -methoden in die Schule einzubringen vermögen. Der Widerruf ihres Beamtenverhältnisses kann aus »irgendwelchen sachlichen Gründen * erfolgen (H. J. Wolff, Verwaltungsrecht II, I962, S. 36 I; vgl. z. B. Ordnung der Laufbahn im Lehramt an höheren Schulen vom April I954 - Amtsblatt [Hessen] S. I 44). Die Abhängigkeit der jungen Assessoren und damit der $Z$ wang, sich althergebrachten Unterrichtsmodellen zu fügen, wird so offenkundig. Wenn es dann in der juristischen Literatur heißr, der Lehrer sei in der Methode seines Unterrichts frei (I. v. Münch, Die pädagogische Freiheit des Lehrers, Deutsches Verwaltungsblatt 1964,789 ff, 795), so kann solche Meinung nur unter Abstrahierung sozialer und gesellschaftlicher Abhängigkeitsverhältnisse aufgestellt werden.

Der ${ }_{\text {Fall }}$ des Assessors Lüdde mag als Einzelfall angesehen werden. Seine Bedeutung gewinnt er vor dem Hintergrund einer Gesellschaft, die oppositionelles politisches Engagement mit rechtlichen Mitteln auszuschalten versucht.

Dieter Hart

\title{
Justiz und NS-Verbrechen
}

Vor kurzem ist der erste Band der insgesame 2r Bände der Serie "Justiz und NS-Verbrechen " erschienen, einer Sammlung der in der Zeit vom 8. 5. 1945 bis zum I. I. 1966 ergangenen rechtskräftigen Urteile der deutschen Land- und Rechtsmittelgerichte in Strafsachen wegen nationalsozialistischer Tötungsverbrechen 1 .

Es erscheint mir nützlich zu sein, den Zweck der Sammlung und die Weise ihres Zustandekommens zu erläutern, insbesondere den wohl etwas merkwürdig anmutenden Umstand zu erklären, daß gerade diese Sammlung, sei es auch unter starker deutscher Beteiligung ${ }^{2}$, an der Universität von Amsterdam vorbereitet wird und bei einem niederländischen Verlag erscheinen muß.

Um den letzten Punkt gleich vorwegzunehmen: Zwar wurde die Bearbeitung von der Alexander-von-Humboldt-Stiftung und wird sie jetzt von der Deutschen Forschungsgemeinschaft und von der niederländischen Forschungsgemeinschaft $* Z$. W. O. subventioniert, ein Druckeostenzuschuß stand und steht jedoch nicht zur Verfügung. Von allen - zuerst in der Bundesrepublik, später auch in den Niederlanden - angegangenen Verlagen war nur einer bereir, das Risiko selbst voll zu übernehmen: die Verlegergemeinschaft "Associated Publishers

1 Gemeint sind damit Mord und Totshlag, ferner Körperverletzung, Freiheitsberaubung, Rechrs beugung, Kriegsverbrechen und Verbrechen gegen die Menschlichkeit, jeweils $\gg$ mit Todesfolgex. $\mathrm{Dabei}$ ist nichr darauf abgestellt, daß es zu einer Verurteilung gekommen ist. Maßgebend für eine Veröffentichung in der Sammlung ist vielmehr der Gegenstand der Anklage. Ferner komme es nicht darauf an, ob die Todesfolge in einem nach dem Tatbestand des betreffenden Strafgesetzes relevanten Kausaizusammenhang mit der strafbaren Handlung steht. Bei einigen Verbrechen könnte ein solcher Zusammenhang auch gar niche festgestellt werden, da das Geriche sich darüber nicht ausläßr, weil der Todeserfolg, z. B. bei den Verbrechen gegen die Menschlichkeir, nicht $z$ den gesetzlichen Tatbestandsmerkmalen gehört.

2 Der Redaktion gehören deutscherseits Prof. Dr. Karl Dietrich Bracher/Bonn und Prof. Dr. Hans Heinrich Jeschede/Freiburg an. Auch Dr. Fritz Bauer hat als Redaktionsmitglied bis zu seinem Tode an den Vorbereitungen starken Anteil genommen. Die Bearbeitung erfolgt maßgeb. lich durch die deutsche Juristin Frau A. L. Rüter-Ehlermana. 
Amsterdam «. Allerdings dürfte das Fehlen einer Subvention nicht immer der einzige Grund für die Absage der deutschen Verlage gewesen sein: ein großer rechtswissenschaftlicher Verlag lehnte die Arbeit ab, obwohl ihm die Ubernahme unter der Bedingung einer Subvention in von ihm zu bestimmender Höhe angeboten worden war.

Welcher Zweck wird nun mit der Veröffentlichung dieser Urteile verfolgt?

Seit 1945 fanden auf dem Territorium der heurigen Bundesrepublik Deutschland Verfahren statt, die nationalsozialistische Straftaten zum Gegenstand hatten. Vom 8. S. I 945 bis zum 3I. I 2. 1967 wurden gegen nicht weniger als 77004 Personen Ermittlungsverfahren durchgeführt. Davon wurden 6 I 92 rechtskräftig verurteilt, gegen s 877 Personen wurde das Verfahren ohne Bestrafung abgeschlossen.

Bei der heutigen Rechtslage werden die Prozesse wegen NS-Gewaltverbrechen, soweit diese den Tatbestand des Mordes erfüllen - alle übrigen Straftaten sind, falls die Verjährung nicht unterbrochen ist, bekanntlich seit spätestens I 960 verjährt - bis in die neunziger Jahre hinein andauern. Am I. I. I968 waren noch gegen I 8935 Personen Verfahren anhängig!

Bereits die geschichtliche Einmaligkeit einer solchen Reaktion der Justiz auf Verbrechen, die in einem vorangegangenen Herrschaftssystem begangen und in seinem Wesen begründer sind, dürfte die Veröffentlichung der Urteile im Interesse der Rechtsgeschichte gerechtfertigt erscheinen lassen, da gerade doch hier dokumentiert wird, wie eine normale Strafjustiz mit nichtnormalen Verbrechen fertig zu werden sich abgemüht hat.

Die Notwendigkeit einer solchen Veröffentlichung zur Erhaltung dieser historisch wertvolien Materialien deurete sich bereits im Laufe der ursprünglich nur für meine privaten Forschungszwedse erfolgten Sammlung der Urteile an, als sich herausstellte, daß einige Urteile, obwohl nachweislich ergangen, nicht mehr vorhanden waren, während andere Urteile, insbesondere solche größerer Bedeutung, die in den ersten Nachkriegsjahren ergangen waren, infolge der schlechten Papierqualität und dem wiederholten Versand schon stark beschädigt, teilweise unleserlich und manchmal sogar im Begriff waren sich buchstäblich aufzulösen. Auch kam die Vermutung auf, u. a. auf Grund der Vermerke auf den Strafakten über die Aufbewahrungsfrist, daß die seit 1958 ergangenen Anordnungen der Länder, Strafakten von Verfahren wegen NS-Verbrechen grundsärzlich als »geschichtlich wertvoll « von der normalen, beschränkten Aufbewahrungsfrist auszusondern und somit ihre Vernichtung zu verhindern, nicht in allen Fällen rückwirkend - zur Anwendung kommen würden.

Dadurch drohten auch die durch die Strafverfahren zutage geförderten historischen Erkenntnisse, die in so manches Ereignis mehr Licht gebracht haben, als es einem Historiker möglich gewesen wäre ${ }^{3}$, der historischen Forschung allmählich und unbemerkt verloren zu gehen.

Den unmittelbaren Anlaß für diese Veröffentlichung bildeten jedoch nicht diese historischen Erwägungen, sondern die heutigen Probleme der Ahndung von NSStraftaten, die anläßlich des 46. Deutschen Juristentages skizziert und mit der dringenden Aufforderung der Wissenschaft verbunden wurden, »die mit den NSGewaltverbrechen verbundenen Fragenkreise gründlich zu untersuchen "t. Die Entschließung der zahlreichen Sachverständigen, die sich auf Einladung der

3 So der historische Sachverständige, Prof. Dr. Hans Buchheim, April r967 im Frankfurter Eurhanasieprozeß ./. Dr. Aquilin Ullrich u. A.

- Verhandlungen des 46. Deutschen Juristentages, Band II, Teil C, Probleme der Verfolgung und Ahndung von nacionalsozialistischen Gewaltverbrechen «. München/Berlin 1967, S. Io. 
Ständigen Deputation des Deutschen Juristentages im April 1966 zu einer Klausurtagung in Königstein zusammengefunden hatten zur Erörterung der mit der Verfolgung und Ahndung der NS-Gewaltverbrechen zusammenhängenden strafrechtlichen und strafprozessualen Problematik, führt in einer Anlage eine ganze Reihe von Problemen auf, die sie selber als einen $»$ Ausschnitt« aus der Fülle der Gesamtproblematik bezeichnet, die jedoch, jedes für sich, intensivste Untersuchungen notwendig machen würden.

Dabei stellte sich sofort die Frage, auf welche Materialien diese Untersuchungen sich stützen sollten. Die Vermutung, daß zumindest die Behandlung der wichtigeren Rechtsfragen in der Höchstrichterlichen Rechtsprechung in der üblichen Weise, in den Sammlungen und Zeitschriften, vorzufinden sei, bewahrheitet sich nicht. Ein Kenner der Materie wie Herbert Jäger hat in seiner 1967 erschienenen bahnbrechenden Studie ${ }^{5}$ darauf hingewiesen, $» d a ß$ bisher nur ganz ausnahmsweise Urteile des Bundesgerichtshofs in Verfahren wegen nationalsozialistischer $\mathrm{Ge}$ waltverbrechen in die Amtliche Entscheidungssammlung aufgenommen oder in den Zeitschriften abgedruckt worden sind " und daß wviele Urteile auch dann unveröffentlicht geblieben sind, wenn sie von grundsätzlicher Bedeutung waren «.

Darüber hinaus kann man sich mit der Auswertung der Höchstrichterlichen Rechrsprechung - auch wenn sie vollständig vorliegen würde - dann nicht begnügen, wenn es sich um eine Problematik handelt, die fast ausschließlich in der Tatsacheninstanz einer endgültigen Lösung zugeführt werden wird, wie dies z. B. bei der auch in der Offentlichkeit stark erörterten Strafzumessung der Fall ist. Es ist nicht ersichtlich, wie die in Königstein angeregte Untersuchung der $»$ Probleme der Strafzumessung bei NS-Gewaltverbrechen, dargestellt im Rahmen einer Analyse bisher ergangener Urteile “ durchgeführt werden kann, wenn die einschlägigen Urteile der Landgerichte nicht zugänglich sind. Diese Landgerichtsurteile sind jedoch, abgesehen von einigen wenigen Ausnahmen ${ }^{6}$, nicht veröffentlicht und sogar für den Forscher kaum vollständig und nicht ohne gewaltigen Zeitverlust zu beschaffen. Herbert Jäger, der sich hauptsächlich auf die erfahrungsgemäß leichter zu beschaffenden Urreile aus den Jahren $1957-1963$ beschränkt hat, berichtet darüber: "Bei der Beschaffung der Urteile wirkte sich nachteilig aus, daß es bisher an einer Regelung über die wissenschaftliche Auswertung gerichtlicher Entscheidungen und Akten leider noch fehlt. Es ist begreiflicherweise für eine solche Untersuchung außerordentlich ungünstig und hinderlich, wenn man auf die Bereitwilligkeit der Staatsanwaltschaften und Gerichte, Urteilsausfertigungen zur Verfügung zu stellen, angewiesen ist, eine Bereitwilligkeir, die zum größeren Teil vorhanden war, in manchen Fällen jedoch fehlte. «

Weit größere Schwierigkeiten bereiter die Sammlung der Urteile aus den Jahren bis I953, während indessen diese weitaus größte Zahl der Urteile nicht außeracht gelassen werden kann. Gerade hier kann es sehr lange dauern, bis eine Ausfertigung beschafft worden ist, z. B. dann, wenn das letzte vorhandene Urteilsexemplar, eine Urschrift, mit der Strafakte versandt, dort, wo sie sein sollte, nicht auffindbar ist, dann völlig unerwartet bei einer dritten Staatsanwaltschaft auftaucht, die allerdings wegen der schlechten Qualität der Urschrift keine Ablichtung und wegen der Uberlastung ihrer Kanzlei keine Abschrift ferrigen kann,

\footnotetext{
${ }^{5}$ Herberr J̈̈ger, Verbrechen unter totalitärer Herrschaft. Olten/Freiburg i. Br., 1967, S. 16.

${ }^{8}$ So sind drei Urteile - der Landgerichte Bonn, Bayreuth und Hanau - veröffentlicht in: Van Dam, H. G., und Giordano, R. (Hrsg.), KZ-Verbrechen vor deutschen Gerichten. Frankfurr/ Main, 2. Aufl., 1966.
} 
so daß das Urteil dazu erst an die Staatsanwaltschaft bei dem zuständigen Landgericht zurückgesandt werden muß.

Sind bereits diese Schwierigkeiten bei der Materialbeschaffung einer als dringend erforderlich bezeichneten Erforschung der Problematik der NS-Verfahren nicht zuträglich, so hiltt auch der private Besitz von Ausfertigungen aller einschlägigen Entscheidungen kaum weiter.

Einmal ist diese über 30000 Seiten umfassende Sammlung mangels jeglichen Registers kaum zugänglich und für die Erforschung eines der Fragenkreise, worauf sich auch ein Forscherteam angesichts der Fülle des Materials wohl beschränken muß, völlig ungeeignet; zum anderen kann eine wissenschaftliche Diskussion mit den Autoren von Studien, die sich auf im Privatbesitz befindliche Urteile stützen, mangels einer allgemeinen Zugänglichkeit dieser Urteile, nicht stattfinden.

Die Praxis ist dementsprechend: einer Fülle von Veröffentlichungen zu den Nürnberger Urteilen stehen nur wenige Arbeiten gegenüber, die sich mit der deutschen Rechtsprechung in Sachen NS-Verbrechen befassen; eine einigermaßen erhebliche wissenschaftliche Diskussion findet nicht statt.

Diese Entwidklung ist um so bemerkenswerter, als gerade die Ahndung dieser Verbrechen, noch ganz abgesehen von der mit dieser Ahndung verbundenen rechrlichen Problematik, keineswegs eine "Vergangenheitsangelegenheit» ist: staatlich organisierte und totalitäre Verbrechen gehören keineswegs der Vergangenheit an, sie sind auch heutzutage von einer bedrüdkenden Aktualität.

Die Redaktionsmitglieder haben sich auf Grund dieser Erwägungen Anfang des Jahres 1967 bereitgefunden, die in den Jahren 1962-1969 am Max-Plandk-Institut für ausländisches und internationales Strafrecht in Freiburg i. Br. und seitdem am Seminarium van Hamel der Universität von Amsterdam für meine privaten Forschungszwecke gesammelten Entscheidungen deutscher Gerichte wegen NS-Tötungsverbrechen, in Form einer mit Erläuterungen und Registern versehenen Quellenveröffentlichung herauszubringen.

Die Redaktion hat die Auswahl- und Bearbeitungsrichtlinien festgelegt und bei ihrer Durchführung die Bearbeiter in umfassender Weise beraten und ihnen geholfen, Schwierigkeiten auszuräumen, wo immer sie auftauchten.

Die Sammlung der Urteile ist nicht dadurch geschaffen worden, daß sie mit List und Tücke den widerstrebenden deutschen Justizbehörden entrungen sind. Im Gegenteil: Die Landesjustizverwaltungen und das Bundesjustizministerium haben sich, sobald ich mit umfassender Unterstützung von Prof. Jescheck, Freiburg, tätig wurde, außerordentlich hilfsbereit gezeigt. Keine einzige angeforderte Entscheidung ist mir vorenthalten worden. Besonders hervorzuheben ist die Hilfe, die von den Staarsanwaltschaften, den eigentlich Leidtragenden meines Forschungsvorhabens, gewährt worden ist. Sie haben seit 1963 den nicht abreißenden Strom von Urteilsanforderungen und Anfragen mit unendlicher Mühe, groBer Hilfsbereitschaft und gleichbleibender Freundlichkeit erledigt. Diese Sammlung ist zu einem großen Teil ihre Arbeit und ihr Verdienst.

Besondere Schwierigkeiten bereitete indessen die Anforderung der Urteile, weil anfänglich niemand wußte, welche Urteile überhaupt ergangen waren.

Bis zum Jahre $196 \mathrm{I}$ fehlte den Justizbehörden der Bundesrepublik eine Ubersicht über die in ihren Geschäftsbereichen seit dem Jahre 1945 wegen NS-Straftaten ergangenen Strafurteile. Noch im Oktober 1960 wurde beim ersten Versuch einer Sammlung des einschlägigen Materials von den Landesjustizverwaltungen übereinstimmend mitgeteilt, daß über die Strafverfahren wegen NS-Verbrechen keine besondere Statistik geführt werde und daß eine nachträgliche Ermittlung der 
Urteile arbeitsmäßig nicht zu bewältigen sei. Im Zusammenhang mit dem Eichmann-Prozeß und dem dadurch stark anwachsenden Interesse der Offentlichkeit an dem Problem der Ahndung der NS-Verbrechen ergriff jedoch der Bundesminister der Justiz im März 196I die Initiative, um zu einem Uberblidk über die wichtigsten Strafverfahren wegen NS-Verbrechen zu gelangen. Er bat zu diesem Zwedk die Landesjustizverwaltungen um die Mitreilung derjenigen Fälle, die bedeutsam erscheinen «, und um eine kurze Beschreibung des Prozeßverlaufs. Die Bearbeitung dieses Ersuchens durch die Landesjustizverwaltungen war jedoch nicht einheitlich und demgemäß waren auch die mitgeteilren Verfahren ihrer Art nach verschieden. Während einige Länder die Wichtigkeit der Fälle nach der Zahl und der zeirgeschichtlichen Bedeutung der Angeklagten, nach der Zahl der Opfer und der Höhe des Strafmaßes beurteilten, teilten andere Länder diejenigen Verfahren mit, die ihnen im Hinblick auf eine von der Bundesregierung vorbereiteten Dokumentation über die Ahndung von NS-Verbrechen als beispielhaft erschienen.

Die auf Grund dieser Meldungen angelegte Kartei, die etwa I650 Verfahren umfaßte, wurde auf Grund einer Genehmigung des Bundesjustizministeriums im Juli 1963 ausgewertet. Da jedoch anzunehmen war, daß die genannte Kartei schon deswegen nicht vollständig sein konnte, weil nur nach den *bedeutsamen Verfahren gefragt worden war und die nach dem März I96I ergangenen Urteile offensichtlich nur vereinzelt gemeldet worden waren, wurden anschließend sämtliche deutsche Fachzeitschriften auf Hinweise auf Verfahren wegen NS-Tötungsverbrechen durchgesehen. Ausgewerter wurden weiter die Berichte und Aufstellungen der Zentralen Stelle der Landesjustizverwaltungen in Ludwigsburg und die in der Bibliothek des Bundesgerichtshofs vorhandene Sammlung sämtlicher Entscheidungen des ehemaligen Obersten Gerichtshofs für die Britische Zone. Seit dem Jahre 1963 wurden ferner die in der deutschen Presse erscheinenden Meldungen über Verfahren wegen NS-Verbrechen gesammelt (monarlich etwa 600). Durch Auswertung dieser Pressemitteilungen wurde eine Vollständigkeir erreicht, die über diejenige der jährlich aufgesteliten behördlichen Ubersichten hinausgeht. Auch durch die Auswertung der ermittelten und inzwischen angeforderten und überlassenen Entscheidungen konnten weitere einschlägige Verfahren erfaßt werden.

Im Zusammenhang mir der Frage der Verlängerung der Verjährungsfrist für Mord bat der Bundesminister der Justiz die Landesjustizverwaltungen am 29. Dezember 1964 um Mitteilung sämtlicher wegen NS-Verbrechen durchgeführten Strafverfahren. Die auf Grund dieses Ersuchens von den Ländern vorgelegten umfangreichen und detaillierten Berichte wurden im Jahre i 965 durchgearbeitet und die dadurch noch zusätzlich ermittelten Entscheidungen - etwa ein Fünftel des jetzt veröffentlichten Materials - bei den Staatsanwaltschaften angefordert. Auch diese Urteile wurden auf Hinweis auf noch nicht bekannte Verfahren durchgesehen.

Die ermittelten Entscheidungen wurden, nachdem die Oberlassung für Forschungszwedke grundsätzlich genehmigt worden war, seit Oktober 1964 den zuständigen Justizbehörden mitgeteilt und von diesen übersandt, wobei von einer Erhebung der üblichen Schreibgebühr, die sich für die ganze Sammlung auf fast I $6000,--D$ DM belaufen hätre, abgesehen wurde.

Die so gesammelten Entscheidungen waren allerdings überlassen worden mit der Auflage, "die Unterlagen vertraulich zu behandeln und ... die Namen der beteiligten Personen nicht mitzuteilen und (die) Angaben nach Möglichkeit so zu fassen, daß von Außenstehenden kein Schluß auf die Identitär der an den Ver- 
fahren beteiligten Personen gezogen werden könne«. Die für eine Veröffentlichung in der Serie »Justiz und NS-Verbrechen * deshalb erforderliche Genehmigung wurde von den Ministern und Senatoren der Justiz erteilt mit der Maßgabe, daß die Namen der Verfahrensbeteiligten durch Anfangsbuchstaben ersetzr werden würden, während allerdings hinsichtlich solcher Personen, die in den betreffenden Verfahren zum Tode oder zu Zuchthausstrafen verurteilt worden waren, eine vollständige Wiedergabe des Namens anheimgegeben wurde.

Die von einem der Landesjustizministerien im Interesse der Wahrung des Persönlichkeitsschutzes der Verfahrensbeteiligten gemachten Vorbehalte, die auf eine Kontrolle der ordnungsgemäßen Durchführung der von der Redaktion ausgearbeiteten Bearbeitungsrichtlinien durch das betreffende Ministerium hinausliefen, haben das Erscheinen des ersten Bandes erheblich verzögert, weil die Redaktion glaubte, auf dieser Basis die Serie nicht herausbringen zu können. Entgegenkommenderweise hat der betreffende Justizminister jedoch später diese Vorbehalte fallen lassen.

Das Zustandekommen des r. Bandes wird überschatter durch den Tod des hervorragenden Redakrionsmitglieds, des Hessischen Generalstaatsanwalts Dr. Fritz Bauer. Dr. Bauer hat, bereits bevor der Gedanke einer Veröffentlichung aufkam, sich bei der Sammlung der Urteile helfend eingesetzt. Seit Februar 1967 gehörte er der Redaktion an, an deren Arbeit er intensiv teilgenommen hat. Den Bearbeirern war er bei der Lösung ihrer im Laufe der Bearbeitung erwachsenen Schwierigkeiten und Probleme mit seinen großen menschlichen und fachlichen Qualitäten ein hochgeschätzter Freund und Ratgeber.

Dr. Bauer hat nie darüber $Z$ weifel gelassen, daß die Tatsache der Veröffentlichung und die Art und Weise, wie sie verwirklicht werden sollte, in seinem Sinne waren.

Ich bin der Hoffnung, daß das jetzt fertiggestellte erste Ergebnis seine Zustimmung gefunden bätte.

C. F. Rüter, Amsterdam 